\title{
Clinical correlation of peripheral CD4+-cell sub-sets, their imbalance and Parkinson's disease
}

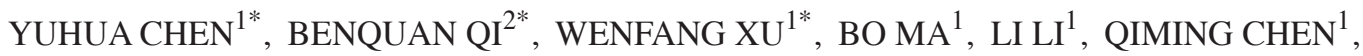 \\ WEIDONG QIAN $^{1}$, XIAOLIN LIU ${ }^{1}$ and HONGDANG QU ${ }^{1}$
}

\begin{abstract}
Departments of ${ }^{1}$ Neurology and ${ }^{2}$ Emergency Internal Medicine, The First Affiliated Hospital of Bengbu Medical College, Bengbu, Anhui 233004, P.R. China
\end{abstract}

Received October 7, 2014; Accepted June 18, 2015

DOI: $10.3892 / \mathrm{mmr} .2015 .4136$

\begin{abstract}
Emerging evidence suggests that the peripheral immune system has an active role in the progression of Parkinson's disease (PD). The finding of T-helper (Th; CD4+) cells infiltrating into the substantia nigra in PD patients demonstrated that Th cells are involved in PD. However, the association between peripheral T-helper cell sub-sets (Th1, Th2, Treg and Th17) and the sub-set balance (Th1/Th2 and Th17/Treg) and PD has remained elusive. In the present study, sixty PD patients of the First Affiliated Hospital of Bengbu Medical College as well as 40 age- and environment-matched healthy individuals were enrolled. The fraction of CD4+ $\mathrm{T}$ cells in the peripheral blood was assessed by automated hematology analysis and its sub-sets (Thl, Th2, Th17, Treg) were quantified by flow cytometry. The results showed that in the PD group, the proportion of Th1 and Th17 cells was increased, while that of Th2 and Treg cells was decreased. Compared with the control group, the Th1/Th2 and Th17/Treg ratios were significantly enhanced, and shifted towards Th1 and Th17, respectively. Furthermore, this Th1-type response (Th1/Th2 balance shifting towards Th1) were associated with motor function scores determined by Unified Parkinson's Disease Rating Scale III (UPDRS-III) scores. However, no correlation was found between the change in the Th17/Treg cell balance (Th17/Treg balance shifting towards Th1) and UPDRS-III scores. These data supported that chronic immune stimulation, specifically CD4+-cell sub-set imbalance, is linked to PD pathobiology and disease severity. CD4+-cell sub-sets and their imbalance may therefore represent novel biomarkers or therapeutic targets for PD.
\end{abstract}

Correspondence to: Professor Hongdang Qu, Department of Neurology, The First Affiliated Hospital of Bengbu Medical College, 287 Changhuai Road, Bengbu, Anhui 233004, P.R. China E-mail: doctorcyh@163.com

*Contributed equally

Key words: Parkinson's disease, CD4+ T cells, T-helper type 1/2 cell balance, T-helper type 17/T-regulatory cell balance

\section{Introduction}

Parkinson's disease (PD) is the second-most common neurodegenerative disease worldwide (1). The typical pathological feature is the abnormal accumulation of $\alpha$-synuclein (SYN) in substantia nigra (SN) dopaminergic neurons, causing the formation of Lewy bodies (LBs) and the degeneration and apoptosis of neurons. To date, the etiology and pathogenesis of abnormal SYN accumulation have remained elusive $(2,3)$. Studies have shown that inflammatory and abnormal immune responses have a crucial role in the occurrence of PD (4-7). These immune-associated types of inflammation exist not only in the brain $(7,8)$, but the peripheral immune system is also thought to contribute to the onset and progression of the neurodegenerative process in PD (9-12). Inflammation in the peripheral immune system is hypothesized to contribute to the onset and progression of the neurodegenerative process observed in PD, due to serum $\alpha \mathrm{SYN}$-specific antibodies (10), and lymphocyte infiltration into the brains of patients with PD (7). In light of these studies, it is indicated that the function of immune cells is essential during inflammation-induced PD.

CD4+ T cells are important immune cells with functions including the identification of viruses, bacteria and other pathogens, and the coordination of other immune cells, directing them to attack these pathogens (13). CD4+ T cells were found to infiltrate into the $\mathrm{SN}$ in $\mathrm{PD}$ patients as well as mouse models of PD (14-17). Naive CD4+ T cells can differentiate into T-helper (Th1, Th2 and Th17) and regulatory $\mathrm{T}$ (Treg) cells in order to execute their functional activities in the immune system. These sub-sets are not differentiated stereotypical cells, but have a certain plasticity $(17,18)$. They affect and restrain each other through the secretion of cytokines, forming a network to maintain homeostasis. An imbalance in the sub-set ratios can cause immune dysfunction and lead to immune damage of organs (19). Studies have shown that circulating CD4+ T-cell sub-sets and sub-set balance may have an active role in the progression of PD $(20,21)$. Only a small number of studies have demonstrated that circulating CD4+ T cell sub-sets are altered in PD patients, however, with conflicting results $(22,23)$.

In the present study, the fractions of Th1, Th2, Th17 and Treg cells which differentiated from CD4+ T cells were assessed in patients with PD. Furthermore, these sub-sets and 
their rations were correlated with Unified Parkinson's Disease Rating Scale III (UPDRS-III) scores of PD patients. The present study indicated that imbalances in CD4+ sub-sets may be utilized as novel biomarkers for PD.

\section{Materials and methods}

Subjects. Blood samples were obtained aseptically by venipuncture from PD patients $(n=60)$ and age-, gender- and environment-matched controls $(n=40)$. Sixty PD patients (32 males and 28 females) received treatment at the First Affiliated Hospital of Bengbu Medical College (Bengbu,China) were enrolled between January 2012 and October 2013. Their age range was 40-74 years with an average age of 61.4 years. In addition, 40 healthy volunteers (average age, 60.8 years; 20 males and 20 females) from the patients' families (spouses) and the First Affiliated Hospital of Bengbu Medical Center, whose age and gender matched those of the PD group, were recruited as the control group. All patients were asked to provide details on their medical history, including time of PD onset, duration, onset process, past history, family history and medication. The relevant scores, as determined by the Unified Parkinson's Disease Rating Scale-III (UPDS-III), were established by specifically trained neurologists. Patients with a history of autoimmune or inflammatory disorders and those receiving chronic immunosuppressive therapy were excluded from the present study.

Written informed consent was obtained from all participants and the study was conducted in accordance with the declaration of Helsinki with approval from the Ethics Committee of Bengbu Medical College (Bengbu, China).

Sample collection. Sterile quantitative blood collection tubes containing EDTA-2 K (BD Vacutainer; BD Biosciences, Franklin Lakes, NJ, USA) were used to draw $8 \mathrm{ml}$ peripheral venous blood. Samples were coded and stored at room temperature until processing, which occurred within $2 \mathrm{~h}$ of collection. A complete blood cell count with differential analysis was performed using an XT-4000i automated hematology analyzer (Sysmex, Kobe, Japan) based on the semiconductor laser fluorescence streaming technology.

\section{Flow cytometric phenotype analysis}

Reagents. Multicolor flow cytometric analysis was performed using a FACSCalibur flow cytometer (BD Biosciences) with BD FACSDiva hardware (BD Biosciences) and CellQuest Pro software (BD Biosciences). Cells were labeled with fluorochrome-conjugated monoclonal antibodies against the following antigens: Anti-human CD8 fluorescein isothiocyanate (FITC) (cat. no. 11-0087), mouse immunoglobulin (Ig)G1 phycoerythrin (PE) (cat. no. 12-4714), anti-human interleukin (IL)-4 PE (cat. no. 12-7049), anti-human interferon (IFN) $-\gamma$ PE (cat. no. 12-7319), IL-17A PE (cat. no. 12-7178), CD3 FITC (cat. no. 1538-03), CD4 FITC (cat. no. 347324), CD25 PE (cat. no. 347643), CD127 peridinin chlorophyll (PerC P)-Cy 5.5 (cat. no. 557938) (all from BD Biosciences). Heparin RPMI 1640 medium, ionomycin mixture, monensin mixture were from MultiScience Lianke Biotech Co., Ltd. (Hangzhou, China). Isotype-matched mouse or rat monoclonal antibodies were used as negative controls.
Th1 and Th2 cell detection. The specific cytokines IFN- $\gamma$ and IL-4 secreted by Th1 and Th2 cells were used to distinguish these two cell types. Anti-CD3 and CD8 labeling with reverse mapping of CD4+ cells was used to prevent propylene glycol methyl ether acetate (PMA)-induced CD4 cell-surface endocytosis. Fresh sterile blood samples $(200 \mu 1)$, heparin anti-coagulant and $200 \mu \mathrm{l}$ RPMI-1640 [MultiScience (Lianke) Biotech Co., Ltd., Hangzhou, China] were added to a $10-\mu$ l PMA $(11 \mu \mathrm{g} / \mathrm{ml}), 8-\mu 1$ ionomycin $(50 \mu \mathrm{g} / \mathrm{ml})$ and $8-\mu l$ monensin $(0.1 \mathrm{mg} / \mathrm{ml})$ working solution [MultiScience (Lianke) Biotech Co., Ltd.]. The mixture was incubated in a $5 \% \mathrm{CO}_{2}$ incubator at $37^{\circ} \mathrm{C}$ for $4-6 \mathrm{~h}$. CD3-FITC and CD8-FITC (BD Biosciences) were added, followed by incubation. The mixture was divided into four aliquots, and to each of them, $100 \mu \mathrm{l}$ stained whole blood was added. Fixative (100 $\mu \mathrm{l}$; BD Biosciences) was added and incubated. Following centrifugation, the supernatant was discarded, and $100 \mu \mathrm{l}$ solubilizing agent (BD Biosciences) was added, followed by mouse IgG1-PE, IFN- $\gamma$-PE, rat IgG1-PE and IL-4-PE and the mixture was incubated in the dark at room temperature for $15 \mathrm{~min}$. Cells were then washed, centrifuged at a speed of $206 \mathrm{x}$ g and the supernatant was discarded. Cells $\left(1 \times 10^{6}\right)$ were resuspended in $0.5 \mathrm{ml}$ phosphate-buffered saline (PBS), then detection of the proportions of CD3 + CD8-IFN- $\gamma+$ cells (Th1 cells) and CD3 + CD8-IL-4+cells (Th1 cells) with a FACS Calibur flow cytometer (BD Biosciences) was performed.

CD4+-cell and Th17-cell detection. Fresh sterile blood samples $(2 \mathrm{ml})$ were mixed with $100 \mu \mathrm{l}$ heparin RPMI-1640 medium [MultiScience (Lianke) Biotech Co., Ltd.]. $5 \mu 1$ $2 \mu \mathrm{g} / \mathrm{ml}$ phorbol ester, $5 \mu \mathrm{l} 50 \mu \mathrm{g} / \mathrm{ml}$ ionomycin and $4 \mu \mathrm{l}$ $5 \mathrm{mg} / \mathrm{ml}$ brefeldin were added, and the cells were incubated at $37^{\circ} \mathrm{C}$ in a $5 \% \mathrm{CO}_{2}$ incubator for $5 \mathrm{~h}$. Fixative $(100 \mu \mathrm{l}$; BD Biosciences) was added and the mixture was incubated in the dark at room temperature for $15 \mathrm{~min}$. Cells were then washed, centrifuged at a speed of $206 \mathrm{x} \mathrm{g}$, and the supernatant was discarded. Solubilizing agent (100 $\mu \mathrm{l}$; BD Biosciences) was added to disrupt the cell membrane. Cells were then stained with allophycocyanine (APC)-conjugated mouse anti-human CD3 (BD Biosciences), PerCP-labeled mouse anti-human CD4 BD Biosciences) and PE-labeled IL-17A-directed monoclonal antibodies (Abcam) or PE-labeled isotype control at $35^{\circ} \mathrm{C}$ in the dark for $15 \mathrm{~min}$. After rinsing twice and centrifugation at a speed of $206 \mathrm{x} \mathrm{g}$, the supernatant was discarded. PBS was added and the samples were examined using the FACSCalibur flow cytometer to determine the proportion of CD3+, CD4+, and IL-17A+ cells.

Treg detection. Tregs were defined as CD3+ CD4+ CD25high CD127diminished (dim), as it has been shown that these cells represent the large majority of forkhead box (Fox) $\mathrm{P} 3+$ Tregs $(24,25)$. In the present study, this definition was used instead of the commonly accepted definition (CD3+ CD4+ CD25high FoxP3+) to avoid excessive cell loss from blood samples during the fixation and washing steps required for FoxP3 staining. Fresh sterile blood $(100 \mu \mathrm{l})$ containing heparin was incubated with APC-conjugated mouse anti-human CD3, FITC-labeled mouse anti-human CD25, PerCP-labeled mouse anti-human CD4 and PE-conjugated mouse anti-human CD127 monoclonal antibodies (all BD Biosciences) and the appropriate isotype controls at $35^{\circ} \mathrm{C}$ in the dark for $15 \mathrm{~min}$. FACS lysis solution (BD Biosciences) was added and the two 
Table I. Complete blood counts and differential counts of PD patients and controls.

\begin{tabular}{|c|c|c|c|c|c|c|c|c|c|c|}
\hline \multirow[b]{2}{*}{ Blood cell count } & \multicolumn{4}{|c|}{ Controls } & \multicolumn{4}{|c|}{ PD patients } & \multirow[b]{2}{*}{ P-value } & \multirow[b]{2}{*}{ t-value } \\
\hline & $\mathrm{n}$ & Mean & $\mathrm{SD}$ & SEM & $\mathrm{n}$ & Mean & $\mathrm{SD}$ & SEM & & \\
\hline Absolute WBC x10 $/ 1$ & 40 & 5.89 & 1.13 & 0.18 & 45 & 6.06 & 1.33 & 0.20 & 0.483 & 0.615 \\
\hline Absolute neutrophils $\times 10^{9} / \mu 1$ & 40 & 3.33 & 0.75 & 0.12 & 45 & 3.82 & 1.14 & 0.17 & 0.030 & 2.306 \\
\hline Neutrophils (\%) & 40 & 56.42 & 5.57 & 0.88 & 45 & 62.58 & 8.98 & 1.33 & 0.003 & 3.841 \\
\hline Absolute lymphocytes $\times 10^{9} / 1$ & 40 & 1.90 & 0.45 & 0.07 & 45 & 1.72 & 0.58 & 0.07 & 0.141 & -1.538 \\
\hline Lymphocyte (\%) & 40 & 31.88 & 4.00 & 0.63 & 45 & 28.93 & 8.19 & 1.22 & 0.001 & -2.143 \\
\hline $\mathrm{CD} 3+\mathrm{CD} 4+(\%)$ & 40 & 38.00 & 3.18 & 0.50 & 45 & 34.96 & 5.19 & 0.77 & $<0.001$ & -3.288 \\
\hline Absolute $\mathrm{CD} 3+\mathrm{CD} 4+\operatorname{count} / \mu \mathrm{l}^{\mathrm{a}}$ & 40 & 719.68 & 175.82 & 27.80 & 45 & 603.51 & 221.91 & 33.08 & 0.071 & -2.652 \\
\hline
\end{tabular}

${ }^{a}$ Absolute CD4+ T-cell count was calculated using the percentage of CD4+ T cells determined by flow cytometric analysis with the absolute lymphocyte count determined by differential analysis. PD, Parkinson's disease; SD, standard deviation; SEM, standard error of the mean; WBC, white blood cells.

Table II. CD4+ T-cell sub-sets and sub-set balance in PD patients and controls.

\begin{tabular}{|c|c|c|c|c|c|c|c|c|}
\hline \multirow[b]{2}{*}{ T-cell sub-set } & \multicolumn{3}{|c|}{ Controls } & \multicolumn{3}{|c|}{ PD patients } & \multirow[b]{2}{*}{ P-value } & \multirow[b]{2}{*}{ t-value } \\
\hline & $\mathrm{n}$ & Mean & $\mathrm{SD}$ & $\mathrm{n}$ & Mean & $\mathrm{SD}$ & & \\
\hline Th1 & 40 & 11.23 & 1.34 & 60 & 17.88 & 6.21 & $<0.001$ & -8.026 \\
\hline Th2 & 40 & 1.82 & 0.19 & 60 & 1.67 & 0.26 & 0.001 & -3.282 \\
\hline Th17 & 40 & 0.52 & 0.16 & 60 & 0.86 & 0.18 & $<0.001$ & -9.798 \\
\hline Treg & 40 & 5.91 & 0.42 & 60 & 3.89 & 0.42 & $<0.001$ & 15.125 \\
\hline Th1/Th2 & 40 & 6.22 & 0.88 & 60 & 10.92 & 4.02 & $<0.001$ & -8.767 \\
\hline Th17/Treg & 40 & 0.09 & 0.03 & 60 & 0.23 & 0.06 & $<0.001$ & -14.645 \\
\hline
\end{tabular}

PD, Parkinson's disease; SD, standard deviation; Th, T-helper cell; Treg, T-regulatory cell.

samples, which were sample treated with all antibodies and IgG1-PE isotype control were incubated for $5 \mathrm{~min}$ in the dark at $35^{\circ} \mathrm{C}$ for $15 \mathrm{~min}$. Cells were then washed and centrifuged at a speed of $206 \mathrm{x} \mathrm{g}$, and the supernatant was discarded. The cells were fixed with $0.5 \mathrm{ml}$ of $1 \%$ paraformaldehyde. Multicolor flow cytometric analysis was performed using the FACSCalibur flow cytometer.

Statistical analysis. Data were analyzed using SPSS 13.0 (SPSS, Inc., Chicago, IL, USA), and graphs were drawn using Graphpad Prism 4 (Graphpad Inc., La Jolla, CA, USA). Comparison between the experimental and control groups was performed using a two-sample $t$-test. Each index comparison between different groups was performed using analysis of variance and a pairwise Student-Newman-Keuls comparison test. $\mathrm{P}<0.01$ was considered to indicate a statistically significant difference.

\section{Results}

Lymphocyte and CD3+CD4+ T-cell frequencies in patients with PD. Increasing evidence suggested that CD4+ T cells have an important role in the pathogenesis of PD (14-16).
Therefore, the numbers of CD4+ T cells in PD patients were determined by flow cytometry. CD4+ T cells are a type of lymphocytes, the latter accounting for $20-40 \%$ of white blood cells (WBC). Thus, the present study first determined the WBC count and the main components of peripheral WBC, neutrophils and lymphocytes. The results in Table I show that neutrophils were enhanced in the PD group, while the absolute lymphocyte value, the lymphocyte proportion of WBCs, the CD4+ T-cell proportion and the absolute CD4+ T-cell number in lymphocytes were decreased in the PD group compared with those in the control group. The CD4+ T-cell proportion and the absolute CD4+ T-cell number in lymphocytes were also higher in the PD group than those in the control group. These results suggested that peripheral blood CD4+ T cells may be involved in the pathogenesis of PD.

PD patients show an enhanced Thl and Th17-type immune response. Naive CD4+ T cells can differentiate into T-helper cells (Th1, Th2 and Th17) and Treg cells, which affect each other. An imbalance can cause immune dysfunction and lead to immune damage of organs (19). Studies have shown that circulating CD4+ T-cell sub-sets and the sub-set balance may have an active role in the progression of PD $(20,21)$. The fact 
A

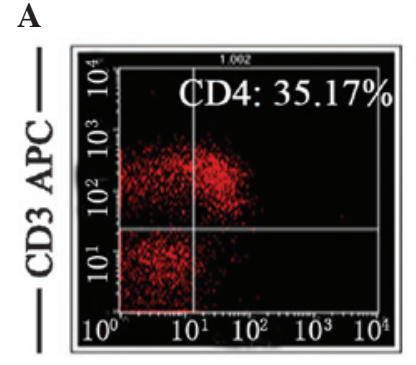

B
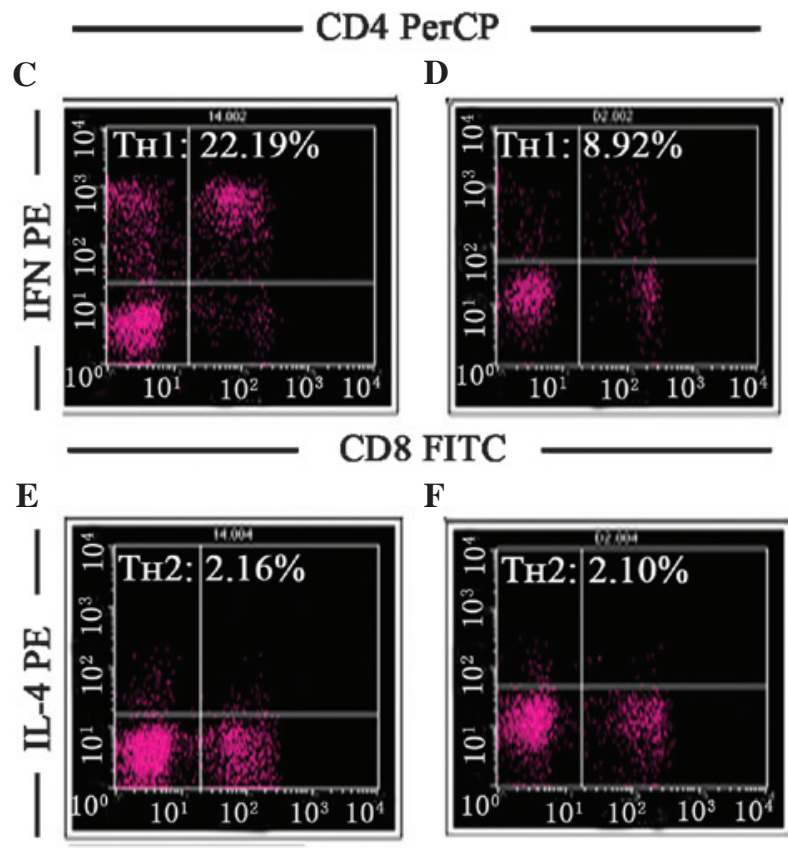

$\mathbf{F}$
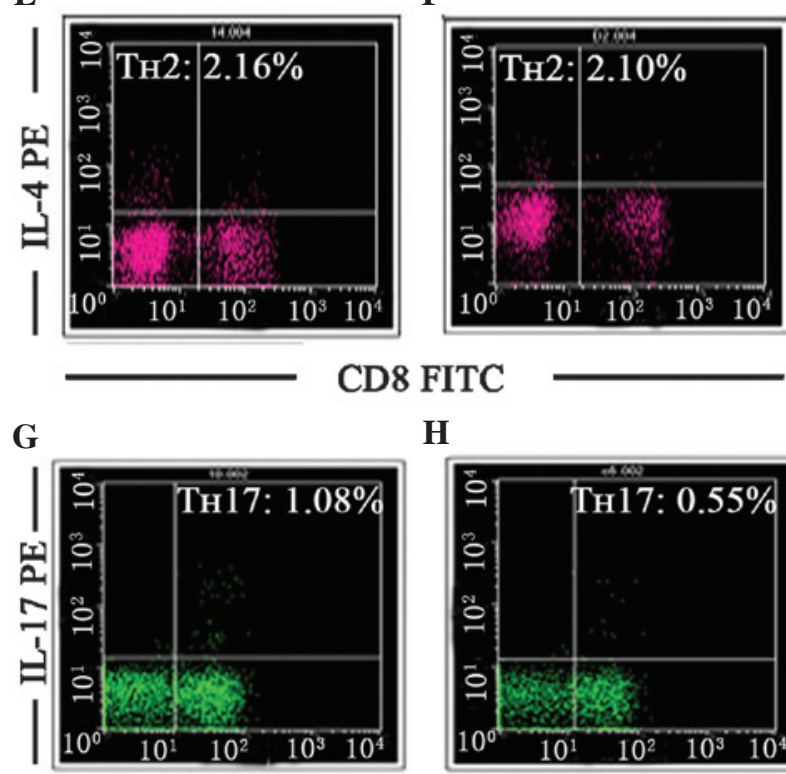

$\mathbf{H}$

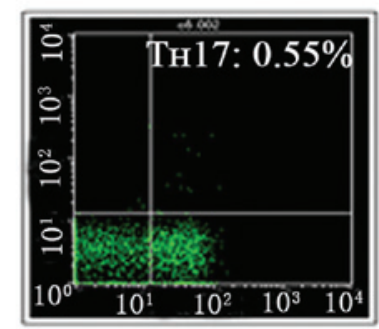

CD4 PerCP

I

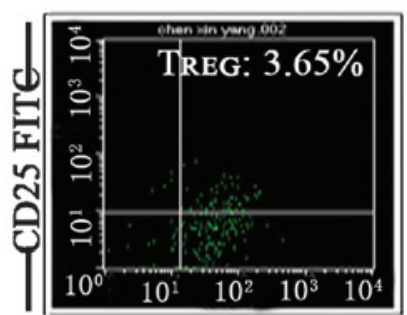

$\mathbf{J}$

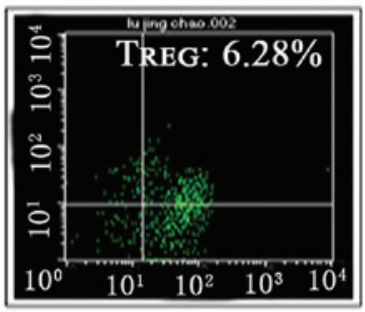

CD127 PE

Figure 1. Gating strategy for flow cytometric analysis of peripheral blood mononuclear cells for the frequency of CD4+ T, Th1, Th2, Th17 and Treg cells. Gating and quantification of (A) CD4+ T-cells in the PD group, (B) CD4+ T-cells in the control group, (C) Th1 cells in the PD group, (D) Th1 cells in the control group, (E) Th2 cells in the PD group, (F) Th2 cells in the control group, $(\mathrm{G})$ Th17 cells in the PD group $(\mathrm{H})$ Th17 cells in the control group, (I) Treg cells in the PD group and (J) Treg cells in the control group. PR, phycoerythrin; FITC, fluorescein isothiocyanate; IL, interleukin; APC allophycocyanin; PerCp, peridinin chlorophyll; PD, Parkinson's disease; Th, T-helper; Treg, T-regulatory. that peripheral blood CD4+ T cells may be involved in the pathogenesis of PD suggested that the occurrence of PD may be correlated with CD4+-cell sub-sets (Th1, Th2, Th17 and Tregs) and sub-set balances (Th1/Th2 and Th17/Treg). To test this hypothesis, the present study examined the numbers of Thl, Th2, Thl7 and Treg cells in the peripheral blood of patients with PD. The ratios of Th1/Th2 and Th17/Treg were then calculated.

To quantify Thl and Th2 cells, the specific cytokines IFN- $\gamma$ and IL-4, which are secreted by Th1 and Th2, respectively, were used to identify those two types of cell. anti-CD3 and CD8 labeling with reverse mapping of CD4+ cells was used to prevent PMA-induced CD4 cell surface endocytosis. To test for Thl7, the specific cytokine IL-17, which is secreted by Th17, was used to distinguish Th17 cells. Tregs were defined as CD3+CD4+CD25high and CD127dim cells. In the present study, this definition was used instead of the 'gold standard' (CD3+ CD4+ CD25high FoxP3+) to avoid excessive cell loss from blood samples during the fixation and washing steps required for FoxP3 staining; studies have shown that $\mathrm{CD} 3+$ CD4+ CD25high CD127dim cells represent the large majority of FoxP3+ Tregs $(24,25)$.

The results showed that Thl and Thl7 cells were obviously enhanced in PD patients group, while the proportion of Th2 and Treg cells significantly decreased (Table II). The Th1/Th2 and Th17/Treg ratios in the PD group were significantly higher than those in the control group, and were shifted towards Th1 and Th17. The enhancement of Th1-type response (the Th1/Th2 balance shifting towards Th1) indicated that the cellular immunity was in the active state and the host cell defended intracellular pathogen infection. This is consistent with the pathological features comprising the abnormal accumulation of SYN in SN dopamine neurons in PD patients. This intracellular abnormal SYN is similar to intracellular pathogens and activates the immune system, leading to the observed Th1-type response. Th17 cells promote inflammation, mainly by producing cytokines, while Tregs inhibit the immune response. Therefore, Th17 and Tregs regulate each other. In the present study, similarly to the Th1-type response, the enhancement of the Th17-type response (Th17/Treg balance shifting towards Th17) also suggested that the inflammation was initiated.

Severity of PD is correlated with CD4+T-cell sub-sets and sub-set balance. To investigate the association between motor dysfunction severity and CD4+ T-cells sub-sets and sub-set balance, the flow cytometric data of the PD group were correlated with the UPDRS-III scores. Three groups were segregated according to UPDRS-III scores of 1-20 ( $n=15)$, $21-30 \quad(n=29)$ and $\geq 31 \quad(n=16)$. Correlation analysis showed that the Th1-type response was associated with motor function scores determined by UPDRS-III. However, no correlation between changes in the Th17/Treg-cell balance and UPDRS-III was identified (Fig. 2, P>0.05).

\section{Discussion}

Inflammation is a defensive reaction against harmful stimuli that can induce a defensive response in the body. In the present study, peripheral blood parameters of PD patients (the general 

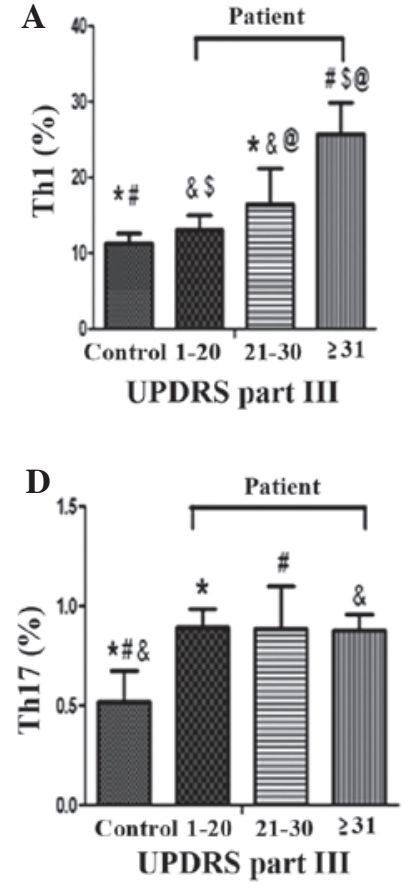
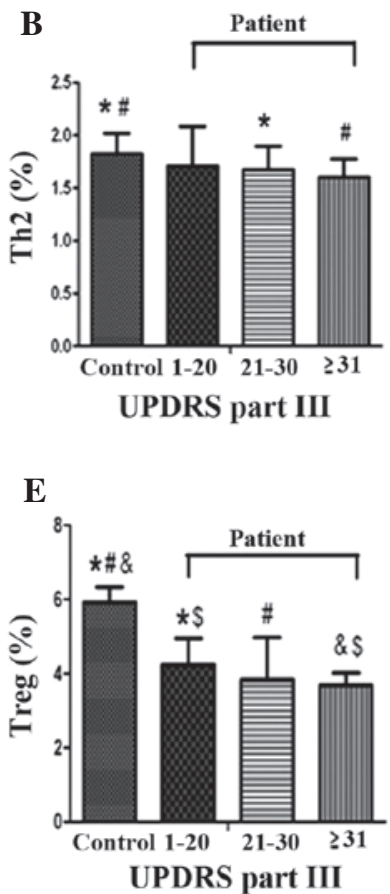
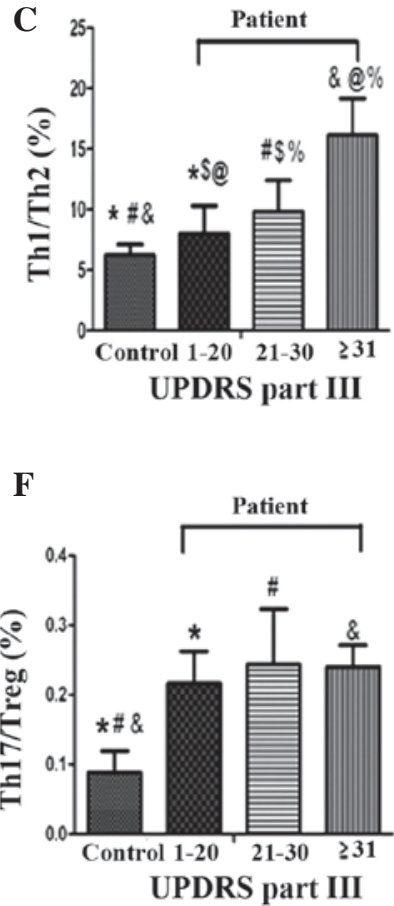

Figure 2. Correlation between (A) Thl-cell fraction, (B) Th2-cell fraction, (C) the Th1/Th2 cell balance, (D) the Thl7-cell fraction, (E) the Treg-cell fraction and $(\mathrm{F})$ the Th17/Treg cell balance and UPDRS-III scores. ${ }^{*} \mathrm{P}<0.01$ for comparison between the control group and $\mathrm{PD}$ group with score $1-20$. ${ }^{\mathrm{P}}<0.01$ for comparison between the control group and $\mathrm{PD}$ group with score $21-30$. ${ }^{\&} \mathrm{P}<0.01$ for comparison between the control group and $\mathrm{PD}$ group with score $\geq 31$. ${ }^{\$} \mathrm{P}<0.01$ for comparison between PD groups with score $1-20$ and score $21-30$. ${ }^{@} \mathrm{P}<0.01$ for comparison between $\mathrm{PD}$ groups with score $1-20$ and score $\geq 31 . \%$. $\mathrm{P}<0.01$ for comparison between PD groups with score 21-30 and score $\geq 31$. PD, Parkinson's disease; UPDRS part III, Unified Parkinson's Disease Rating Scale III scores; Th, T-helper; Treg, T-regulatory.

parameters of WBCs, as shown in Table I) were within the normal range (4-10x10 $/ 1)$, however, the proportion of neutrophils in PD patients was high. In PD patients, the proportion of CD4+ cells in lymphocytes was low, but the difference from the healthy control group was not statistically significant. Further study confirmed that the distribution of CD4+-cell sub-sets in PD patients was obviously abnormal, which indicated alterations in the immune response in PD patients. This result was consistent with the findings of previous studies, which suggested that peripheral immunological challenges and chronic inflammatory diseases influence the pathogenesis and progression of PD (26-29). These studies indicated that CD4+ cells have an important role in the development of PD.

In the immune system, CD4+ $\mathrm{T}$ lymphocytes are important immune cells, and all CD4+ T cells can be classified into Thelper (Th1, Th2, Th17) and regulatory T (Treg) cell sub-sets. Through the secretion of cytokines, they affect and restrain each other, and form a network to maintain homeostasis for maintaining the normal immune function. $\mathrm{Mu}$ et al (30) first presented the concept that functional imbalance of Th1-, Th2-, Th17- and Treg-cell sub-sets leads to multiple sclerosis. The present study confirmed the changes in the distribution of CD4+-cell sub-sets in PD patients. However, if the above-mentioned network balance is broken, it will lead to a disease (30).

The present study reported that the Th1-cell population was increased and the Th1/Th2 balance was shifted towards Th1 in PD patients. The normal proportion of Th1 cells among CD4+ cells is $10-15 \%$ (the proportion of the subsets refers to the total CD4+-cell population and these reference values are derived from the application of flow cytometry, which was performed for Th1/Th2 detection). The normal range of Th1 was $11.8-25.8 \%$ and the normal range of Th2 was $0.8-2.4 \%$. In the present study, Th1-cell populations in the PD group and control group were 17.88 and $11.23 \%$, respectively; therefore, the Th1-cell population in the PD group was higher than the measured value of the control group. The normal value for the Th2-cell population is $0.8-2.4 \%$. In the present study, Th2-cell populations in the PD group and control group were 1.82 and $1.67 \%$, respectively. Although the Th2 population in the PD group was decreased, the difference from the control group was no statistically significant and values were within the normal range. The Th1/Th2 balance shifted towards the Th1 cells, indicating an enhanced Th1-type response in PD patients. Previous studies indicated that Th1 cells mediate cell immunity through secretion of cytokines and have an important role in removing intracellular microbial infections (13). Th2 cells mediate the humoral immune response through the secretion of cytokines. Under normal conditions, the immune function of Th1 and Th2 cells is in a state of dynamic equilibrium. Once the equilibrium is disturbed, normal immune function cannot be maintained with the emergence of various associated diseases $(18,19)$. It is known that the Th1/Th2-cell imbalance is closely associated with infectious diseases, autoimmune diseases and cancer. The Th1-type response is associated with the enhanced immune defense of a host against viral and intracellular pathogen infections $(19,31,32)$. The results of the present study indicated that the Th1-type response was enhanced in PD patients, and PD was based mainly on cell-mediated immunity. This is consistent with the 
pathological features of PD, comprising the abnormal accumulation of SYN in SN dopamine neurons (3). This accumulated SYN in the affected neurons has a similar effect to that of intracellular pathogens, thereby activating the immune system and leading to a Th1-type response and a shift in the Th1/Th2 balance towards Th1. As long as SYN is accumulated in the SN dopamine neurons, it continues to activate the immune system, and the resulting excessive or prolonged immune response leads to PD progression and loss of dopamine neurons. The more the cells are affected, the more severe the disease, and the more marked the Th1-type response and the shifting of the Th1/Th2 balance. Therefore, the present study assessed the correlation between Th1, Th2, the Th1/Th2 balance and UPDRS-III scores in patients with PD. UPDRS-III is the most commonly used assessment of disease severity, based on PD motor symptoms. The correlation analysis showed that in PD patients, Th1 and Th1/Th2 were associated with the motor function scores determined by UPDRS-III. PD patients with UPDRS-III scores of 30 or higher had increased Th1 and Th1/Th2, indicating more severe motor symptoms of PD and the presence of a correlation between the Th1 response and the severity of PD.

The present study also found that Th17 cells were increased and Tregs were decreased in PD patients. The normal proportion of Tregs in human peripheral blood is $5-10 \%$ (33); in the present study, Tregs were reduced to $4.12 \%$ in PD patients and the Th17/Treg balance was shifted towards Th17. Previous studies have shown that Th17 cells promote inflammation mainly through production of cytokines, and are primarily associated with mucosal inflammation $(34,35)$. Normally, Th17 cells and Tregs restrain each other, and there is a Th17/Treg balance, similar to the Th1/Th2 balance. The disturbance of the Th17/Treg balance is a key factor in numerous inflammatory and autoimmune diseases $(34,36)$. In the present study, the increased Th17-cell population in PD patients indicated enhanced PD-associated inflammation, while reduced Tregs in PD patients suggested weakened inhibition of inflammation. This further confirmed not only the existence of an excessive inflammatory response, but also weakened immune inhibition in PD patients. The latter caused the relative enhancement of inflammation, which is responsible for the aggravation of the inflammatory damage to tissues and organs in PD. However, the present study found no significant correlation between the proportion of Th17 cells and the UPDRS-III schore in patients with PD. This imay be because UPDRS-III may not fully reflect the severity of PD, as it only considers motor function. The clinical diagnosis is primarily based on motor symptoms of PD, and therefore, the most commonly used assessment of disease severity is the UPDRS-III score, which is based on motor symptoms. However, in recent years, with the deeper understanding of $\mathrm{PD}$, non-motor symptoms in PD patients also received increased attention $(37,38)$. Numerous studies have shown that $\mathrm{PD}$ is a multicentric neurodegenerative process that also affects neuronal structures outside the central nervous system in the SN $(13,39)$. Studies have identified abnormal accumulation of SYN in olfactory mucosa and the colon, and in the clinic, PD patients generally present with symptoms including the loss of smell and constipation. These symptoms can appear prior to or after the motor symptoms, and therefore, it is unreasonable to exclusively consider motor symptoms to assess the degree of PD.
A preliminary study by our group found that non-specific inflammation in the colonic mucosa, which is associated with long-term inflammation-induced colonic transit constipation, is present in PD patients. The proportion of Th17 cells and Treg cells as well as the Th17/Treg ratio were found to be correlated with constipation of Parkinson's disease patients (results not shown) (40). Th17 cells are closely associated with mucosal inflammation, and Treg cells can be transformed into Th17 cells in the mucous membrane (35), which may explain the present findings that Th17 cells, Treg cells and Th17/Treg were not correlated with the severity of motor symptoms in PD patients.

Experiments addressing the phenotype of pathogenic CD4+T-cells have shown that Th1 and Th17-cells autoreactive cells are important for the promotion of neuronal loss in PD (41). Conversely, other T-cell subsets, such as Tregs-cells and Th2-cells, could contribute to microglial acquisition of an anti-inflammatory phenotype and to promote neuronal protection $(41,42)$.

In conclusion, the results of the present study indicated that chronic immune stimulation, specifically the imbalance of the Th1/Th2 CD4+-cell sub-set, is linked to PD pathobiology and disease severity. The Th1/Th2 equilibrium and its imbalance may therefore represent a novel biomarker or therapeutic target for PD.

\section{Acknowledgements}

The present study was supported by the key projects of Anhui Provinal Department of Education (no. KJ2014A163), the China Postdoctoral Science Foundation (no. 20090461139) and the National Natural Science Foundation of China (no. 81001457).

\section{References}

1. Dorsey ER, Constantinescu R, Thompson JP, et al: Projected number of people with Parkinson's disease in the most populous nations, 2005 through 2030. Neurology 68: 384-386, 2007.

2. Mori F, Nishie M, Kakita A, Yoshimoto M, Takahashi H and Wakabayashi K: Relationship among alpha-synuclein accumulation, dopamine synthesis, and neurodegeneration in Parkinson disease substantia nigra. J Neuropathol Exp Neurol 65: 808-815, 2006.

3. Croisier E, Moran LB, Dexter DT, Pearce RK and Graeber MB: Microglial inflammation in the parkinsonian substantia nigra: Relationship to alphaa-synuclein deposition. J Neuroinflammation 2: 14, 2005.

4. Stevens CH, Rowe D, Morel-Kopp MC, Orr C, Russell T, Ranola M, Ward C and Halliday GM: Reduced T helper and B lymphocytes in Parkinson's disease. J Neuroimmunol 252: 95-99, 2012.

5. Umemura A, Oeda T, Tomita S, Hayashi R, Kohsaka M, Park K, Sugiyama $\mathrm{H}$ and Sawada H: Delirium and high Fever are associated with subacute motor deterioration in Parkinson disease: A nested case-control study. PLOS One 9: e94944, 2014.

6. Noelker C, Morel L, Osterloh A, Alvarez-Fischer D, Lescot T, Breloer M, Gold M, Oertel WH, Henze C, Michel PP, et al: Heat shock protein 60: An endogenous inducer of dopaminergic cell death in Parkinson disease. J Neuroinflammation 11: 86, 2014.

7. More SV, Kumar H, Kim IS, Song SY and Choi DK: Cellular and molecular mediators of neuroinflammation in the pathogenesis of Parkinson's disease. Mediators Inflamm 2013: 952375, 2013.

8. Bakay RA, Boyer KL, Freed CR and Ansari AA: Immunological responses to injury and grafting in the central nervous system of nonhuman primates. Cell Transplant 7: 109-120, 1998.

9. Luk KC, Kehm VM, Zhang B, O'Brien P, Trojanowski JQ and Lee VM: Intracerebral inoculation of pathological $\alpha$-synuclein initiates a rapidly progressive neurodegenerative $\alpha$-synucleinopathy in mice. J Exp Med 209: 975-986, 2012. 
10. Maetzler W, Apel A, Langkamp M, Deuschle C, Dilger SS, Stirnkorb JG, Schulte C, Schleicher E, Gasser T and Berg D: Comparable autoantibody serum levels against amyloid- and inflammation-associated proteins in Parkinson's disease patients and controls. PLOS One 9: e88604, 2014.

11. Hamza TH, Zabetian CP, Tenesa A, Laederach A, Montimurro J, Yearout D, Kay DM, Doheny KF, Paschall J, Pugh E, et al: Common genetic variation in the HLA region is associated with late-onset sporadic Parkinson's disease. Nat Genet 42: 781-785, 2010.

12. Melief J, Koning N, Schuurman KG, Van De Garde MD, Smolders J, Hoek RM, Van Eijk M, Hamann J and Huitinga I: Phenotyping primary human microglia: Tight regulation of LPS responsiveness. Glia 60: 1506-1517, 2012.

13. Shannon KM, Keshavarzian A, Dodiya HB, Jakate S and Kordower JH: Is alpha-synuclein in the colon a biomarker for premotor Parkinson's disease? Evidence from 3 cases. Mov Disord 27: 716-719, 2012.

14. Hirsch EC and Hunot S: Neuroinflammation in Parkinson's disease: A target for neuroprotection? Lancet Neurol 8: 382-397, 2009.

15. Brochard V, Combadière B, Prigent A, Laouar Y, Perrin A, Beray-Berthat V, Bonduelle O, Alvarez-Fischer D, Callebert J, Launay JM, et al: Infiltration of CD4+lymphocytes into the brain contributes to neurodegeneration in a mouse model of Parkinson disease. J Clin Invest 119: 182-192, 2009.

16. González H, Contreras F, Prado C, Elgueta D, Franz D, Bernales S and Pacheco R: Dopamine receptor D3 expressed on CD4+ T cells favors neurodegeneration of dopaminergic neurons during Parkinson's disease. J Immunol 190: 5048-5056, 2013.

17. Zhou L, Chong MM and Littman DR: Plasticity of CD4+ T cell lineage differentiation. Immunity 30: $646-655,2009$.

18. Wan YY: Multi-tasking of helper T cells. Immunology 130 $166-171,2010$

19. Mu L, Sun B, Kong Q, Wang J, Wang G, Zhang S, Wang D, Liu Y, Liu Y, An H, et al: Disequilibrium of T helper type 1, 2 and 17 cells and regulatory $\mathrm{T}$ cells during the development of experimental autoimmune myasthenia gravis. Immunology 128 : e826-e836 2009

20. Nie K, Zhang Y, Gan R, Wang L, Zhao J, Huang Z, Tang H and Wang L. Polymorphisms in immune/inflammatory cytokine genes are related to Parkinson's disease with cognitive impairment in the han chinese population. Neurosci Lett 541: 111-115, 2013.

21. Sanchez-Guajardo V, Annibali A, Jensen PH and Romero-Ramos M: $\alpha$-Synuclein vaccination prevents the accumulation of parkinson disease-like pathologic inclusions in striatum in association with regulatory $\mathrm{T}$ cell recruitment in a rat model. J Neuropathol Exp Neurol 72: 624-645, 2013.

22. Saunders JA, Estes KA, Kosloski LM, Allen HE, Dempsey KM, Torres-Russotto DR, Meza JL, Santamaria PM, Bertoni JM, Murman DL, et al: CD4+ regulatory and effector/memory T cell subsets profile motor dysfunction in Parkinson's disease. J Neuroimmune Pharmacol 7: 927-738, 2012.

23. Niwa F, Kuriyama N, Nakagawa M and Imanishi J: Effects of peripheral lymphocyte subpopulations and the clinical correlation with Parkinson's disease. Geriatr Gerontol Int 12: 102-107, 2012.

24. Fazekas de St Groth B, Zhu E, Asad S and Lee L: Flow cytometric detection of human regulatory $\mathrm{T}$ cells. Methods Mol Biol 707: 263-279, 2011

25. McClymont SA, Putnam AL, Lee MR, Esensten JH, Liu W, Hulme MA, Hoffmüller U, Baron U, Olek S, Bluestone JA, et al: Plasticity of human regulatory $\mathrm{T}$ cells in healthy subjects and patients with type 1 diabetes. J Immunol 186: 3918-3926, 2011.
26. Pott Godoy MC, Ferrari CC and Pitossi FJ: Nigral neurodegeneration triggered by striatal AdIL-1 administration can be exacerbated by systemic IL-1 expression. J Neuroimmunol 222: 29-39, 2010

27. Hasegawa Y, Inagaki T, Sawada M and Suzumura A: Impaired cytokine production by peripheral blood mononuclear cells and monocytes/macrophages in Parkinson's disease. Acta Neurol Scand 101: 159-164, 2000.

28. Brodacki B, Staszewski J, Toczyłowska B, Kozłowska E, Drela N, Chalimoniuk M and Stepien A: Serum interleukin (IL-2, IL-10, IL-6, IL-4), TNF alpha and INF gamma concentrations are elevated in patients with atypical and idiopathic parkinsonism. Neurosci Lett 441: 158-162, 2008

29. Reale M, Iarlori C, Thomas A, Gambi D, Perfetti B, Di Nicola M and Onofrj M: Peripheral cytokines profile in Parkinson's disease. Brain Behav Immun 23: 55-63, 2009.

30. Mu L, Sun B, Kong Q, Wang J, Wang G, Zhang S, Wang D, Liu Y, Liu Y, An H, et al: Disequilibrium of T helper type 1,2 and 17 cells and regulatory $\mathrm{T}$ cells during the development of experimental autoimmune myasthenia gravis. Immunology 128 (1 Suppl): e826-e836, 2009.

31. Hawkins RD, Larjo A, Tripathi SK, Wagner U, Luu Y, Lönnberg T, Raghav SK, Lee LK, Lund R, Ren B, Lähdesmäki H and Lahesmaa R: Global chromatin state analysis reveals lineage-specific enhancers during the initiation of human T helper 1 and T helper 2 cell polarization. Immunity 38: 1271-1284, 2013.

32. Olson NC, Sallam R, Doyle MF, Tracy RP and Huber SA: $\mathrm{T}$ helper cell polarization in healthy people: Implications for cardiovascular disease. J Cardiovasc Transl Res 6: 772-786, 2013.

33. Wan YY and Flavell RA: How diverse - CD4 effector T cells and their functions. J Mol Cell Biol 1: 20-36, 2009.

34. Homey B: After TH1/TH2 now comes Treg/TH17: Significance of T helper cells in immune response organization. Hautarzt 57: 730-732, 2006 (In German)

35. Sakaguchi S, Ono M, Setoguchi R, Yagi H, Hori S, Fehervari Z, Shimizu J, Takahashi T and Nomura T: Foxp3+ CD25+ CD4+ natural regulatory $\mathrm{T}$ cells in dominant self-tolerance and autoimmune disease. Immunol Rev 212: 8-27, 2006.

36. Chen Z, Lin F, Gao Y, Li Z, Zhang J, Xing Y, Deng Z, Yao Z, Tsun A and Li B: FOXP3 and ROR $\gamma \mathrm{t}$ : Transcriptional regulation of Treg and Th17. Int Immunopharmacol 11: 536-542, 2011.

37. Charles PD, Esper GJ and Davis TL: A definition of "on": Patient diaries compared to the UPDRS. Parkinsonism Relat Disord 5: 99-101, 1999

38. Longhi M, Ricciardi G, Tommasi G, Nicolato A, Foroni R, Betrolasi L, Beltramello A, Moretto G, Tinazzi M and Gerosa M: The role of 3T magnetic resonance imaging for targeting the human subthalamic nucleus in deep brain stimulation for Parkinson Disease. J Neurol Surg A Cent Eur Neurosurg 76: 181-189, 2015.

39. Braak H, Sastre M, Bohl JR, de Vos RA and Del Tredici K: Parkinson's disease: Lesions in dorsal horn layer I, involvement of parasympathetic and sympathetic pre- and postganglionic neurons. Acta Neuropathol 113: 421-429, 2007.

40. Chen Y, Yu M, Liu X, Qu H, Chen Q, Qian W, Wei D, Xu W, $\mathrm{Ma} \mathrm{B}$ and $\mathrm{Wu} \mathrm{W}$ : Clinical characteristics and peripheral $\mathrm{T}$ cell subsets in Parkinson's disease patients with constipation. Int J Clin Exp Pathol 8: 2495-2504, 2015.

41. Reynolds AD, Stone DK, Hutter JA, Benner EJ, Mosley RL, Gendelman HE: Regulatory T cells attenuate Th17 cell-mediated nigrostriatal dopaminergic neurodegeneration in a model of Parkinson's disease. J Immunol 184: 22612271.

42. Appel SH: CD4+ T cells mediate cytotoxicity in neurodegenerative diseases. J Clin Invest 119: 13 15, 2009. 